\title{
Modelo de rehabilitación integral
}

\author{
Mercedes del Pilar Valero Pérez.
}

\begin{abstract}
Resumen
El propósito de este articulo es dar a conocer el modelo de rehabilitación integral siendo el que logra hasta nuestros dias el mavor acercamiento como parte de la solución al complejo mundo del usuario con discapacidad, enfatizando en los aspectos multidimensionales que hacen parte de las necesidades axiológicas existenciales del ser humano, desde las habilidades físicas que facilitan las actividades básicas cotidianas y las de la vida diaria. las emocionales que retoman la personalidad, autoestima, relaciones afectivas y manejo de dificultades entre otros, hasta los trazos sociales que marcan el aislamiento, el cambio de rol laboral, familiar, social y recreativo.

El modelo es facilitador de la autonomía. la integración, el reconocimiento de capacidades y derechos humanos, permitiendo apoyos intrínsecos y extrínsecos por parte de la comunidad. los profesionales en salud y las políticas gubernamentales, llevando al individuo rehabilitado a la reinserción familiar; social, y laboral dentro del contexto y medio ambiente en el cual se ha desempeñado.
\end{abstract}

Es importante anotar que a través de todas las épocas se han formulado muchos modelos los cuales se hará remembranza dentro del articulo analizando sus aspectos positivos y negativos ya que permitieron en su momento el favorecimiento de la persona con discapacidad, y muchos aún estan vigentes.

Palabras clave: discapacidad, rehabilitación. modelos en discapacidad. modelo de rehabilitación integral.

\begin{abstract}
The purpose of this article is to present the Model of Integral Rehabilitation, which by the present time. has the best approach as part of the solution to the handicapped user's complex world. This model makes emphasis on the multidimensional aspects, such as physical abilities which make easier the daily basic activities and those of the daily life, the emotional abilities which take back, the personality, self esteem, affective relationships and handing of the difficulties, and the social traces which mark the isolation, the role change in the job, the fanty and recreational issues. All the previous aspects are part of the human being's axiological and existential needs.

The model makes the autonom: the integration, and the recognition of capabilities and human rights easier: this allows the existence of intrinsic and extrinsic support. on the part of the community: health professionals and govermmental policies, taking the rehabilitated individual to the reintegration to the familiar; social, and work world, within the context and environment the individual has alwass lived in.

It is important to write down that through all the times, many models have been formulated, vou will find in this article a remembrance of them, taking their negative and positive aspects into account. These models allowed the benefit of the handicapped person, at their moment, and some of them are currently used.
\end{abstract}

* Fisioterapeuta. Universidad del Rosario. Hospital Militar Central. Especialista en Docencia e Investigación Universitaria y Administración Hospitalaria. Docente Escuela Colombiana de Rehabilitación. 


\section{Tendencias en los modelos de rehabilitación}

\section{Transición desde el modelo médico al integral}

Es importante conocer la transición de los modelos de discapacidad y explorar dentro de ellos el aporte y la tendencia que marcaron dentro de su época histórica. Viniendo desde el modelo tradicional, el médico, el social así como otros que de alguna manera han sido cimiento para llegar al modelo de Rehabilitación Integral (Selman, 2002).

La primera se relaciona con el conflicto entre las y los profesionales en salud que se identifican con el modelo médico y las personas con discapacidad que se identifican con el modelo social, creando su propio movimiento criticando el modelo médico de la discapacidad y demandando mayor participación en las decisiones (Basnett, 2001).

La segunda trata de adiciones sociales derechos humanos y tecnología, reflejándose esta situación en instrumentos como la Ley Estadounidense de Discapacidad (Americans with Disabilities Act 1990), la propuesta para la Convención Internacional de Naciones Unidas sobre los Derechos de las Personas con Discapacidad (United Nations Convention on the Rights of Persons with Disabilities y la Cumbre Mundial sobre la Sociedad de información (World Summit on Information Society.

La tercera tendencia es la investigación en Rehabilitación, que está obligada a mostrar evidencia de resultados, mostrando efectividad y eficacia de la tecnología en la asistencia a las personas con discapacidad (Fuhrer, 2001).

La cuarta tendencia es la lucha de las y los administradores de programas de bienestar social por hacer programas de beneficios solventes, que permitan cada vez la atención a más personas con discapacidad. Por tal motivo en algunos países se están adoptando una mezcla de políticas de bienestar social, derechos civiles y otros elementos para incluir los temas de la discapacidad (Van Oorscot y Hvinden, 2001; Zeiter, 2002).

Y finalmente la quinta tendencia está relacionada con la rehabilitación integral partiendo de la concepción de la discapacidad orientada al reconocimiento de los derechos humanos, la autonomía y capacidad de interactuar dentro de su ambiente y contexto, favoreciendo la inclusión social permitiendo llevar una vida digna y equitativa. Busca combatir la pobreza, considerándola como una barrera para la ejecución de los programas en discapacidad especialmente en los países en desarrollo, teniendo en cuenta que es allí donde están ubicadas la mayoría de personas con discapacidad.

Este modelo emerge de la comunidad internacional básicamente Estados Unidos y Japón quienes hacen grandes esfuerzos políticos, sociales, en capacitación, investigación y tecnología, obteniendo como objetivo final la inclusión social, no sólo la rehabilitación funcional. En la actualidad la tendencia es que se centre el manejo de la discapacidad en las necesidades individuales facilitando la inclusión social; sin perder de vista que el modelo tradicional de discapacidad es muy utilizado en muchas culturas asegurando varios autores que se debe a la ausencia de infraestructuras científicas y de salud, creando así el clima para que las políticas en salud sean producto de la religión y la cultura (Barners y Mercer, 2003; Coleridge, 1993; Ingstad y Whyte, 1995).

\section{Modelos en rehabilitación}

A lo largo de este artículo se expondrán seis modelos en discapacidad, considerándose el conocimiento base, los roles y las relaciones, los parámetros espaciales, temporales y el sesgo. Sin dejar de apostar al aporte que de alguna manera ha servido para crecer dentro de la rehabilitación. 


\section{Modelo tradicional:}

Bases: conocimientos, opiniones y prácticas determinadas por la cultura, organización social y religión (Barnes y Mercer, 2003).

Roles: se habla del desempeño de las personas con discapacidad que van desde participantes a desafortunados (Coleridge, 1993).

Parámetros: los conocimientos objetivos y basados en la ciencia no están asociados con el modelo.

Sesgo: el relativismo cultural.

Aporte: genera reflexión respecto a resolver la paradoja de qué hacer con las personas con discapacidad. En la antigüedad simplemente se les mataba, considerándolos como una carga para su tribu.

\section{Modelo médico:}

Bases: radican en opiniones y prácticas científicas típicamente conocimiento médico y de salud.

Roles: la persona con discapacidad asume el papel de paciente con duración corta o larga.

Parámetros: se tiende a las políticas de la institucionalización con el apoyo de la comunidad, las actitudes profesionales y sociales.

Sesgo: en este modelo la percepción biológica y médica son las válidas dejando a la discapacidad y los factores sociales alejados de su conocimiento.

Aportes: salud y bienestar promoviendo la experimentación algunas veces con buenas prácticas otras no: cuestiona dificultad en propósitos sociales accesibilidad y participación (Gabard y Martín, 2003).

\section{Modelo social}

Bases: conocimientos provenientes de la experiencia, opiniones y prácticas de las personas con discapacidad (Albrech Bury, 200) 1; Barnes y Mercer, 2003). Ubica el problema dentro de la sociedad, no dentro del individuo con discapacidad, concibiendo ésta como la diversidad en función o el resultado de la discriminación en las políticas, prácticas, investigaciones y en la educación.

Roles: Se asumen múltiples roles, dentro de ellos el principal es el defensor de los derechos humanos, se enfatiza la participación plena en la educación, el empleo y la vida ciudadana.

Parámetros: Se expresan dentro de la filosofía de la vida independiente contando siempre con una fuerte organización de la comunidad de las personas con discapacidad.

Sesgos: Limitar las causas de la discapacidad principalmente a las políticas sociales y ambientales.

Aportes: Se enfatiza por los derechos individuales, permitiendo posteriormente luchar por los cambios económicos y sociales que abarcan a los países en desarrollo (Albrech, Seelman y Bury, 200) I; Barnes y Mercer, 2003).

\section{Modelo biopsicosocial:}

Bases: integración de los componentes médico y social, abordando la discapacidad como un problema dentro de la sociedad y no exclusivo de la persona afectada.

Roles: participación en los contextos ambientales capaces de influir positiva o negativamente sobre la persona con discapacidad, con el apoyo de la comunidad.

Parámetros: en este modelo se presenta el ser humano como el eje central tomando elementos biológicos, psicológicos y las condiciones sociales. 
Sesgos: falta integración en los aspectos ambientales y sociales.

Aportes: aborda la discapacidad acercándose al concepto integrador.

\section{Modelo de rehabilitación basado en la comunidad:}

Bases: El problema de la discapacidad no está limitado únicamente a la persona, también hace partícipe a la familia, la comunidad, la sociedad y la cultura en general aparece hacia 1994.

Parámetros: Alternativa para dar una atención integral y aprovechamiento a los recursos personales, comunitarios y del Estado.

Roles: El actor principal es el individuo desempeñando su papel desde la prevención de la discapacidad, la rehabilitación primaria, secundaria y terciaria, hasta su inclusión social.

Sesgos: Son difíciles de encontrar ya que ha sido un método con excelentes resultados aplicado en diferentes países latinoamericanos entre ellos Colombia, México y Bolivia entre otros.

Aportes: Según la Organización Internacional del Trabajo (1999), la rehabilitación basada en la comunidad es un programa que pertenece a la comunidad representada por el gobierno, debiendo formar parte constitutiva de la política social, educativa y sanitaria a cualquier nivel.

Entonces es importante reconocer que estos nuevos modelos son integradores, haciendo énfasis en los elementos propios del individuo, sus necesidades y habilidades, para la integración de los componentes y generar un cambio en el lenguaje; la Organización Mundial de la Salud propone un cambio en la concepción de la discapacidad expresada en la Clasificación Internacional de la Funcionalidad CIF aprobada en mayo de 2001 .
Siendo su objetivo principal brindar un lenguaje unificado y estandarizado dentro de un marco conceptual para describir la salud y sus estados, teniendo en cuenta:

\section{Aspectos relevantes:}

- Funcionamiento

- Discapacidad

- Salud

\section{Dimensión:}

- Funcionamiento y discapacidad

- Factores contextuales

\section{Componentes:}

- Funciones y estructuras corporales

- Actividades y participación

- Factores ambientales

- Factores personales

- Funciones: son las funciones fisiológicas de los sistemas corporales (incluyendo las funciones psicológicas)

- Estructuras: son las partes anatómicas del cuerpo, tales como los órganos, extremidades y sus componentes.

Dentro de la Clasificación Internacional de la Discapacidad es importante tener en cuenta algunos términos que permanentemente vamos a utilizar para expresar la connotación de la discapacidad ellos son:

Deficiencia: son los problemas en las funciones o estructuras corporales.

Actividades: es el desempeño de una tarea por parte de un individuo.

Limitación: son dificultades que un individuo puede tener en el desempeño de las actividades.

Participación: es el acto de involucrarse en una situación vital. 
Restricción: Son problemas que el individuo puede experimentar al involucrarse en situaciones vitales (estudio-dinero).

Al haber reconocido el significado de estos términos y sumarlos podemos decir que la deficiencia más la limitación más la restricción llevan a la discapacidad, de allí la importancia de su aplicación dentro de los modelos.

\section{Modelo ecológico (Schalock)}

\section{(Bronfenbrenner 1989)}

Bases: emerge de la psicología con una visión holística favoreciendo el abordaje de la problemática de la discapacidad con el ánimo de mejorar la calidad de vida de la población.

Roles: ubica al individuo en estado de discapacidad con su experiencia única dentro de su medio ambiente dando el sentido de referencia desde la perspectiva individual.

Parámetros: está constituido por cuatro componentes (proceso, contexto, tiempo y persona).

Sesgo: los factores de experiencias pasadas en el usuario ejercen una influencia única y particular en cada persona.

Aportes: crea sistemas, denominados microsistemas significando éste las características propias del individuo, el mesosistema refleja la comunidad y el macrosistema que refleja las influencias sociales, políticas y culturales.

Luego de la revisión de los diferentes modelos podemos concluir que han dado grandes aportes en su momento y que parte de ellos nos llevan al Modelo Integral de Rehabilitación.

\section{Modelo de rehabilitación integral}

Este modelo es una alternativa para brindar una atención integral aprovechando los recursos per- sonales, comunitarios y del Estado. Con una visión transformadora con la posibilidad de tener una vida digna y equitativa desde la inclusión, el apoyo, la autodeterminación y las capacidades para desarrollarse como persona autosuficiente. Por ello es importante hacer una revisión exhaustiva desde sus principios, perspectivas, sistemas y componentes.

\section{Perspectiva sistémica}

Procesos en el que el uso combinado y coordinado de medidas médicas, terapéuticas, educativas y vocacionales que ayuda a los individuos discapacitados a alcanzar los más altos niveles funcionales posibles y a integrarse a la sociedad.

\section{- Rehabilitación:}

Proceso de duración limitada, y controlado con objetivos definidos encaminados a permitir que una persona con deficiencias alcance un nivel físico, mental y/o social óptimo.

\section{Proporciona:}

- Medios para modificar la vida.

- Crea medidas encaminadas a compensar una función motora.

- Facilita ajustes o reajustes sociales.

\section{Sistema abierto}

- Es aquel que interactúa e intercambia energía con su medio y cuyos objetivos se establecen en función de un sistema jerárquicamente superior.

Permite:

Interdependencia de factores tales como:

- Persona con limitaciones.

- Familia.

- Comunidad.

- Condiciones del entorno socioeconómico y cultural.

- Intercambio interinstitucional. 
Permitiendo el abordaje de la rehabilitación con una visión de integralidad y concepción de interdisciplinariedad.

\section{Rehabilitación integral}

Sistema abierto que la sociedad establece para integrar progresivamente a algunos de sus miembros a la vida productiva.

\section{Favorece:}

- Comprender a la persona con limitación física (individual-social).

- Orienta procesos adecuándose a las necesidades.

- Promueve y facilita el conocimiento del individuo y su medio garantizando responder a exigencias de nuevos sistemas.

- Permite información de retorno, autorregulación, dinamismo y flexibilidad.

\section{Objetivos}

- Definir y entender la dimensión de la problemática que se aborda.

- Identificar procesos para dar respuesta a la problemática.

- Establecer las interrelaciones existentes entre los distintos procesos.

- Conocer y manejar trinomio (persona con limitaciones-entorno- $\mathrm{RH}$ ).

- Identificar los recursos necesarios para dar cumplimiento a los objetivos.

\section{Componentes:}

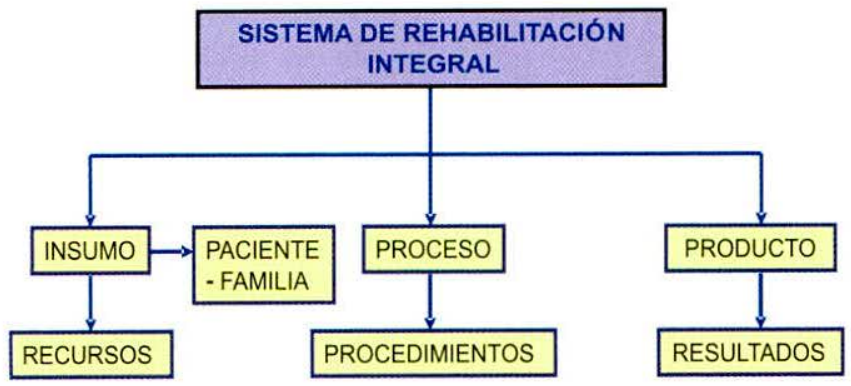

Figura 1
- Insumo: persona con limitaciones y su familia.

\section{- Recursos:}

Filosóficos

Humanos

Materiales

Información

- Procesos- Procedimientos

- Producto- Resultados

Todos estos componentes están dentro de un contexto socioeconómico, e interactúan entre sí dentro del sistema de rehabilitación integral permitiendo autonomía y equiparación de oportunidades para las personas con discapacidad.

\section{Componente insumo}

\section{INSUMO: PERSONA CON LIMITACIÓN Y SU FAMILIA}

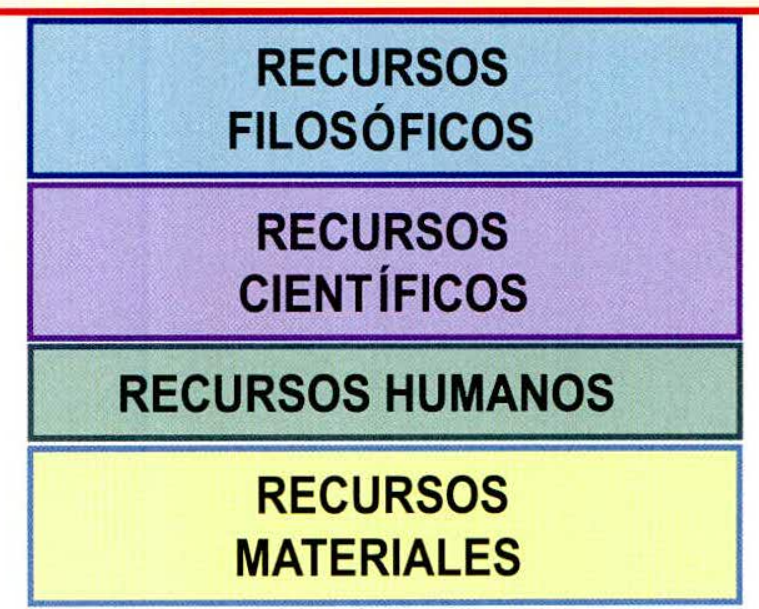

Figura 2

Explicita al ser humano como eje central desde su propia concepción y de la sociedad, tomando esto como un recurso filosófico. Aprovecha los recursos científicos como conocimientos teóricos y técnicos, al recurso humano como proveedor de los servicios de salud; contando con los recursos materiales apoyados en la provisión financiera. 


\section{Componente procesos}

Etapas

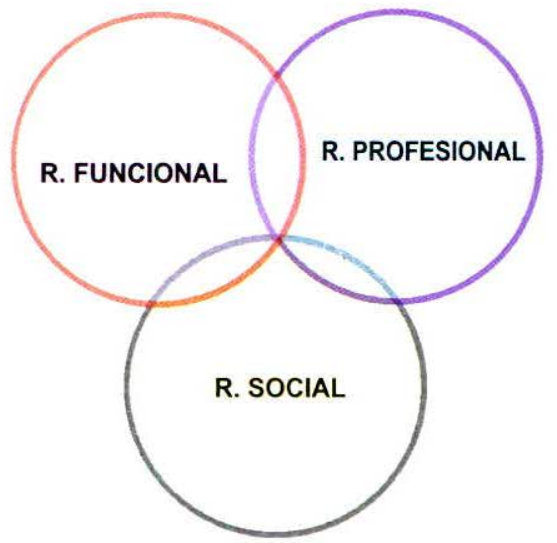

Figura 3

Se basa en el logro de la rehabilitación funcional, cumplida dicha fase se pasará a la profesional y posteriormente a la inclusión social

\section{- Rehabilitación funcional}
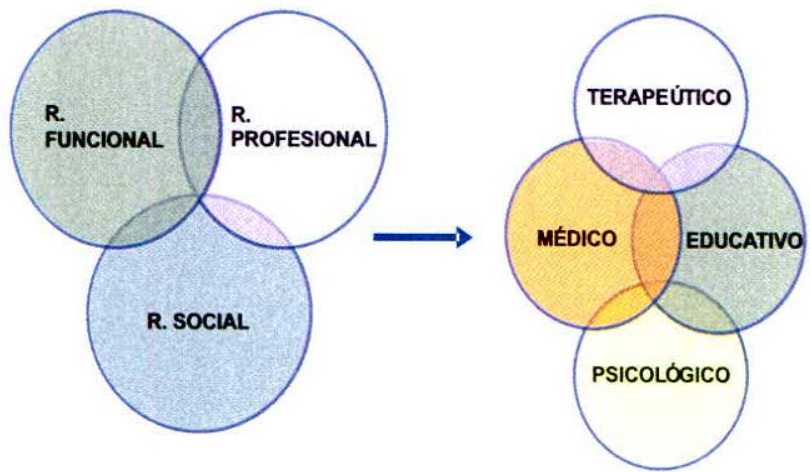

Figura 4

Promueve el logro de la máxima capacidad física, mental y psicológica, determinada por un equipo interdisciplinar de manera oportuna y continua.

\section{Rehabilitación profesional}

Con ello se logra el mayor acercamiento a la inclusión social, haciendo un gran énfasis en la orientación, formación, adaptación y seguimiento favoreciendo su propia autonomía. (Véase Figura 5 )

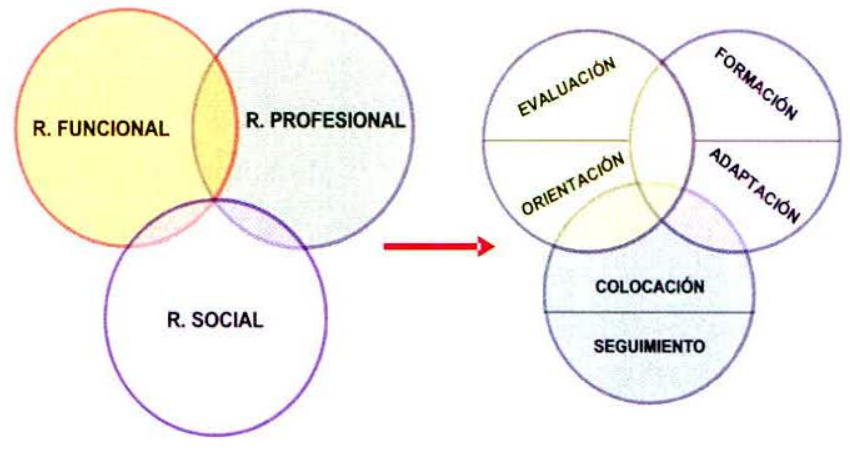

Figura 5

\section{Rehabilitación social}

Producto final de la rehabilitación integral determinando su eficiencia mediante el cumplimiento de objetivos y el favorecimiento al individuo con discapacidad.

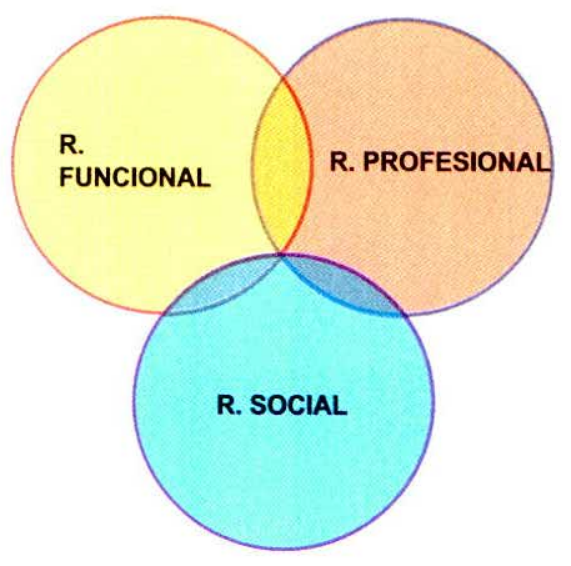

Figura 6

La rehabilitación social está constituida por:

- Acciones

- Instituciones

- Familia

- Comunidad

- Gobierno 


\section{Componentes}

- Procedimientos: es el componente que se apoya en lo tecnológico, administrativo y social. Como facilitadores del manejo del entorno. Deben permanentemente acompañar a las diferentes fases de la rehabilitación para optimizar el producto final. (Véase Figura 7 ).

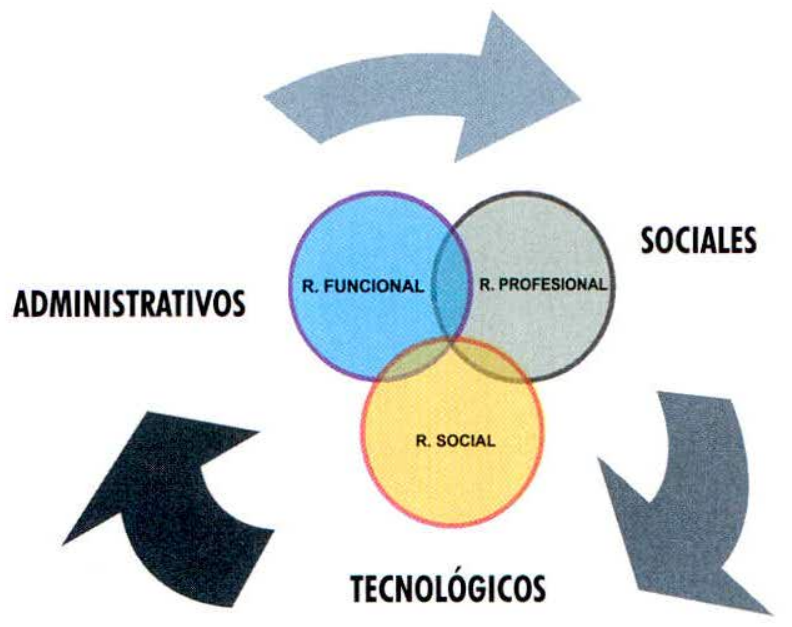

Figura 7

- Informe de gestión: se interpreta como el cumplimiento de objetivos propuestos en el modelo.

- Producto: persona y familia rehabilitada.

- Resultado: integración al medio sociolaboral. (Véase Figura 8 )
- Retroalimentación: mecanismo de control.

- Variables internas.

- Variables externas.

Y se controla:

- La inclusión dentro de los niveles de atención.

- Garantizar ajustes sujeto-entorno.

Con la anterior revisión se han tocado todos los tópicos que revisten la fundamentación, aplicación y control del modelo de rehabilitación integral.

Considero importante en este artículo hacer relevancia a la fisioterapia como una de las disciplinas que ha generado grandes aportes a la rehabilitación integral.

La fisioterapia es la ciencia de la salud que se ocupa del movimiento corporal humano a través de todas sus etapas, desde la edad pediátrica a la geriátrica, promoviendo la salud y previniendo la enfermedad, facilitando la recuperación, habilitación y rehabilitación dentro de los diferentes niveles en salud.

De igual manera ha participado activamente en los procesos y cambios en los modelos de rehabilitación a través de todas las épocas, propendiendo por lograr beneficios motrices facilitadores de la integración e inclusión social de la persona con

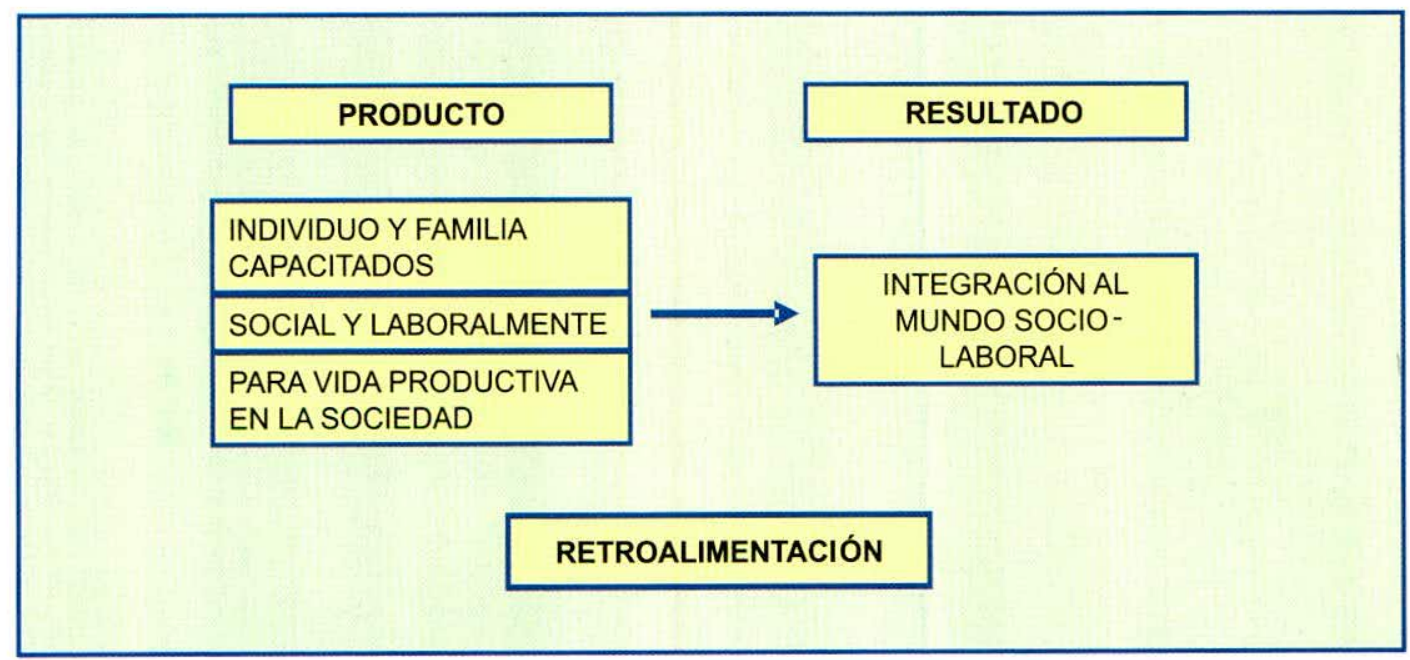

Figura 8 
discapacidad, minimizando su secuela y favoreciendo la funcionalidad.

Teniendo en cuenta que la gran mayoría de lesiones que llevan a la discapacidad son causadas por alteraciones en el sistema nervioso, cabe anotar que la fisioterapia fundamenta su intervención en los modelos de control motor. Ellos han sido tomados en grados variables de la neurofisiología. la biomecánica y la psicología conductual, estas tres ciencias contribuyen a las teorías tradicionales y contemporáneas del desarrollo y el aprendizaje motor.

\section{Antecedentes}

- Modelo REFLEJO

- Modelo JERÁRQUICO

- Modelo SISTEMAS

Se fundamenta en el control motor. La facilitación de la plasticidad cerebral, y el último se orienta a las ganancias funcionales a ejecución de tareas.

De igual manera cobra gran importancia en la actualidad el manejo del condicionamiento físico, como presupuesto indispensable para el desempeño motor y optimización de las ganancias funcio- nales, que posteriormente van a favorecer a la rehabilitación integral.

Recibido: agosto 2006 Aceptado: septiembre 2006

\section{Referencias bibliográficas}

Manual Básico de Rehabilitación Profesional, Grupo Latinoamericano de Rehabilitación Profesional, Edición I. 1991.

Manual de Rehabilitación en Salud.

GLARP. Plan de desarrollo 1987-1990, Bogotá. 1987.

Naciones Unidas, Programa de Acción Mundial para las Personas Lesionadas, Nueva York. 1983.

Stokes M. 2000h. Rehabilitación neurología, Editorial Harcourt Barcelona.

Céspedes GM. "La nueva cultura de la discapacidad y los modelos de rehabilitación".

Organización Mundial de la Salud - OMS. Clasificación Internacional del Funcionamiento y la Discapacidad -CIF- Ginebra, 2000.

Seelman C. Tendencies in the rehabilitation and disability: transition from a medical model to a model of integration. International disability news and views. Issue \#26 June September, 2004. 